Proc. Indian Acad. Sci. (Chem. Sci.), Vol. 112, No. 1, February 2000, pp. 63-72

(C) Indian Academy of Sciences

\title{
Pulsed laser excitation of phosphate stabilised silver nanoparticles
}

\author{
JYOTIRMAYEE MOHANTY, DIPAK K PALIT, LAXMINARAYAN \\ V SHASTRI and AVINASH V SAPRE* \\ Radiation Chemistry and Chemical Dynamics Division, Bhabha Atomic \\ Research Centre, Trombay, Mumbai 400 085, India \\ e-mail: cdscd@magnum.barc.ernet.in
}

MS received 26 August 1999; revised 18 December 1999

\begin{abstract}
Laser flash photolysis studies were carried out on two types of silver nanoparticles prepared by $\gamma$-radiolysis of $\mathrm{Ag}^{+}$solutions in the presence of polyphosphate as the stabiliser. Type I silver nanoparticles displayed a surface plasmon band at $390 \mathrm{~nm}$. Type II silver nanoparticles showed a $390 \mathrm{~nm}$ surface plasmon band with a shoulder at $550 \mathrm{~nm}$. On photoexcitation in the surface plasmon band region, using 35 picosecond laser pulses at $355 \mathrm{~nm}$ and $532 \mathrm{~nm}$, the type II solutions showed transient bleaching and absorption signals in the $450-900 \mathrm{~nm}$ region, which did not decay appreciably up to 5 nanoseconds. These transient changes were found to get annealed in the interval $t$ where $5 \mathrm{~ns}<t<100 \mathrm{~ns}$. Extended photolysis of the nanoparticle solutions with repetitive laser pulses resulted in a decrease in the values of the average particle size which were measured by employing the dynamic light scattering technique.
\end{abstract}

Keywords. Pulsed laser excitation; silver nanoparticles; surface plasmon band; sodium polyphosphate stabilizer.

\section{Introduction}

Nanoparticles, representing the transition region between individual atom/molecule and bulk material have been attracting considerable research input, owing to some unique physical and chemical properties which they exhibit. Colloids and nanoparticles of silver are one of the most widely studied and well characterised metal systems due to the high stability of the silver nanoparticles $(\mathrm{SNP})^{1}$. The silver nanoparticles give transparent aqueous solutions characterised by a strong surface plasmon band (SPB) at $\approx 390 \mathrm{~nm}$ in the optical absorption spectrum ${ }^{2}$. The presence of the SPB gives a convenient handle for the spectroscopic investigations on the metal nanoparticles. The position and shape of SPB of SNPs is strongly dependent on their size, chemical environment of the dielectric media and the nature of the species adsorbed on particle surfaces ${ }^{3-5}$. SNPs also have large electric fields ${ }^{3}$. Metal NPs exhibit excellent photochemical activity due to their high surface/volume ratio and unusual electronic properties ${ }^{6-10}$. Photochemical effects on SNPs have been investigated by a number of workers ${ }^{6-10}$. Henglein and coworkers ${ }^{5,6}$ have observed the photochemical dissolution of SNPs when photoejected electrons are

${ }^{*}$ For correspondence 
scavenged by $\mathrm{N}_{2} \mathrm{O}$. The excitation of SPB of silver and gold NPs has been studied recently by the femtosecond (fs) transient absorption technique ${ }^{8,9}$. These results show that plasmon resonance on photoexcitation is created in $\sim 150 \mathrm{fs}$ and subsequent relaxation processes occur by electron-phonon interactions and phonon cooling mechanism via phonon-solvent interactions in 2 and $\sim 40$ picoseconds (ps) respectively. Transient absorption studies of Ahmadi and coworkers show that the SPB bleaches and a new broad and weak absorption appears at $>600 \mathrm{~nm}$ for gold NPs, with both absorptions decaying in the ps time-scale ${ }^{9}$. Kamat et al have prepared silver NPs by the chemical reduction method and excited with $18 \mathrm{ps}$ pulses at $355 \mathrm{~nm}$ and observed the bleaching of the SPB with rise in the absorption in the red region which was attributed to the photoejection of electrons ${ }^{10}$. They interpret their results in terms of breaking the bigger NPs $(40-60 \mathrm{~nm})$ into smaller ones $(5-20 \mathrm{~nm})$. Wavelength dependence of size selectivity for fragmentation was also seen by them. The weak broad absorption band at $>600 \mathrm{~nm}$ has been attributed by these workers to hydrated electrons $\left(e^{-}\right.$aq $)$. Similar fast relaxations by electron-phonon coupling have also been seen at metal films and metal-quartz interfaces ${ }^{11}$. Recently some work has also been reported on the shaping of the NPs by laser irradiation by Takami et al $^{12}$.

The present study is carried out with the following considerations. Nanoparticles prepared in a liquid environment would be generally associated with other ionic or neutral species present in the form of a stabilizer, complexing agent, etc. Surface modifications of silver particles by different additives have been studied in the past ${ }^{13}$. It is conceivable that the method of preparation of the nanoparticles could influence the relaxation process following the photoexcitation of the particles in the SPB. Roberti et al have prepared SNP by reduction of $\mathrm{AgNO}_{3}$ with $\mathrm{NaBH}_{4}{ }^{8}$. Kamat et al have used the method of reduction of $\mathrm{AgClO}_{4}$ by sodium citrate ${ }^{10}$. We generated SNP by gamma-irradiation of aqueous $\mathrm{AgClO}_{4}$ solutions using polyphosphate as the stabilizer ${ }^{14-17}$. The polyphosphate was shown to be somewhat unique as the stabilizer, in that in addition to stabilizing the metallic clusters of silver, showing the normal SPB absorption, it could also generate under certain experimental conditions, silver clusters of low nuclearity with optical absorptions below $380 \mathrm{~nm}$, having stability up to $\sim 1 \mathrm{~h}$. It was of interest to study transient effects following the photoexcitation of polyphosphate stabilized SNPs, employing our flash photolysis system with $35 \mathrm{ps}, 355 / 532 \mathrm{~nm}$ laser pulses. In the present work, we also studied the effect of laser irradiation, with repetitive pulses, on the long-term changes in the particle size of the SNPs.

\section{Experimental}

SNPs were prepared in aqueous solutions of $\mathrm{AgClO}_{4}$ containing 2-propanol $(\mathrm{PrOH})$ and sodium polyphosphate $\left[\left(\mathrm{NaPO}_{3}\right)_{6}:(\mathrm{PP})\right]$ using the technique of $\gamma$-radiolysis ${ }^{14-17}$. A cobalt60 gamma-source with a dose rate $12 \mathrm{~Gy} / \mathrm{min}$ was used for this purpose. The ground state absorption spectra were recorded with a Shimadzu model 160-A UV-visible spectrophotometer. Characterisation of particles was carried out using a Brookhaven Instruments model BI-900 particle size analyser based on the principle of dynamic light scattering (DLS). Picosecond flash photolysis studies were carried out using either the 2nd or 3rd harmonic $(10 \mathrm{~mJ}$ at $532 \mathrm{~nm}$ and $2 \mathrm{~mJ}$ at $355 \mathrm{~nm})$ output of a Nd-YAG laser having $35 \mathrm{ps}$ pulse duration. Transient absorptions were monitored using the pump and probe technique with the continuum, generated in the range $450-900 \mathrm{~nm}$ by focussing residual $1064 \mathrm{~nm}$ light on to a cell of $\mathrm{H}_{2} \mathrm{O}: \mathrm{D}_{2} \mathrm{O}(1: 1)$, as the probe. The spectra were 
recorded by a dual diode array based optical multichannel analyser ${ }^{18-20}$. Solutions were deoxygenated by $\mathrm{N}_{2}$-bubbling and flowed through a suprasil quartz flow cell $(1 \mathrm{~cm} \times 1 \mathrm{~cm})$ and $(1 \mathrm{~cm} \times 0.2 \mathrm{~cm})$ during the flash photolysis experiments. Nanosecond flash photolysis experiments in nanosecond time domain have been carried out using the same picosecond $\mathrm{Nd}$ : YAG laser for excitation and a tungsten filament lamp as the analysing light source coupled with a Bausch and Lomb monochromator, photomultiplier (Hamamatsu, R-428) and a digital oscilloscope (TDS-540, Tektronics) ${ }^{20}$.

\section{Results and discussion}

\subsection{Preparation and characterisation of Ag nanoparticles}

When $\mathrm{N}_{2}$-purged aqueous solutions of $\mathrm{AgClO}_{4}$ containing $0.1 \mathrm{mM}$ PP and $1.0 \mathrm{mM}$ PrOH were irradiated by $\gamma$-rays at different times, the optical density (OD) at $390 \mathrm{~nm}$ due to SPB increased, reached saturation and then slightly decreased. The radiation dose was optimized to give enough OD at the desired wavelengths $(355 / 532 \mathrm{~nm})$ to carry out flash photolysis experiments. Two $\mathrm{Ag}^{+}$ion concentrations and irradiation times were optimised ((I) $0 \cdot 1-0 \cdot 2 \mathrm{mM} \mathrm{Ag}^{+}, 10 \mathrm{~min}$ and (II) $0.5 \mathrm{mM} \mathrm{Ag}{ }^{+}, 20 \mathrm{~min}$ ) and these are henceforth referred to as type I and type II solutions. The absorption spectra of the surface plasmons obtained in these solutions are given in figures $1 \mathrm{a}, \mathrm{b}$, respectively. Spectra from goldenyellow coloured type I solutions are nearly Gaussian without shoulder and are comparable to those reported by Roberti $e t \mathrm{al}^{8}$.

Type II solutions displayed reddish-brown colour and had a shoulder in the SPB at $\sim 550 \mathrm{~nm}$ in addition to the absorption maximum at $390 \mathrm{~nm}$. In the absence of oxygen

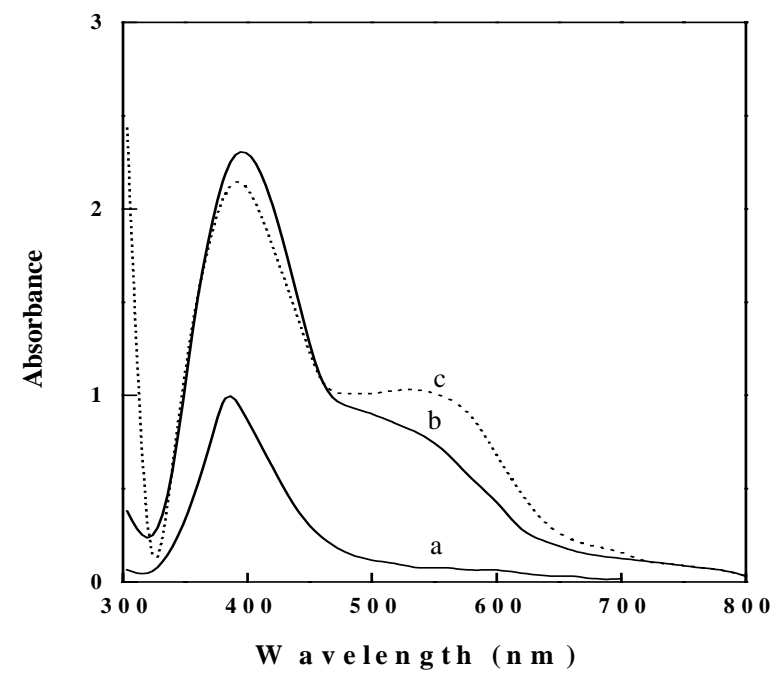

Figure 1. Ground state optical absorption spectra of silver nanoparticles produced on $\gamma$-radiolysis of aqueous solutions. The absorbance values are normalised for path length $=1.0 \mathrm{~cm}$. Initial composition of solutions and duration of gamma irradiations: (a) $0.1 \mathrm{mM} \mathrm{AgClO}, 0.1 \mathrm{mM}$ polyphosphate and $1 \mathrm{mM}$ iso-propanol. $10 \mathrm{~min}$ irradiation (type I solution); (b) $0.5 \mathrm{mM} \mathrm{AgClO} 4,0.5 \mathrm{mM}$ polyphosphate and $5 \mathrm{mM}$ iso-propanol, $20 \mathrm{~min}$ irradiation (type II solution); (c) addition of 10\% (v/v) acetone to the radiolytically prepared type II solution (b). 
they were stable for 4-5 days. When exposed to ambient air the reddish-brown solutions were stable for at least a day. It may be mentioned here that the experimental conditions were somewhat critical for the stability of the solutions. It was found that the reddishbrown solutions turned pale yellowish-green and ODs at $550 \mathrm{~nm}$ decreased at higher $\gamma$ doses. The yellowish-green solutions were found to be unstable and precipitation started in a few hours. Skillman and Berry have measured the absorption spectra of SNP produced in gelatin by photographic exposures and concluded that only one band $(\sim 390 \mathrm{~nm})$ is apparent for nearly spherical particles ${ }^{21}$. Mode splitting occurs for elongated particles with one band moving towards the red region and the other slightly towards the blue. For average axial ratio $(\mathrm{a} / \mathrm{b}) \sim 2.5$ they observed that the red band appears at $\sim 800 \mathrm{~nm}$. On comparison with these results, it can be extrapolated in the present case that for type II solutions the $\mathrm{a} / \mathrm{b}$ ratio is $\sim 2^{21,22}$. All the experiments were carried out on freshly prepared SNPs.

Particle size determinations were carried out in terms of volume average size employing the dynamic light scattering (DLS) technique. The solutions were filtered through $0 \cdot 2$ micron size filters to eliminate dust particles or microcrystals thrown out of the solution, if any, before taking the DLS measurements. Before the $\gamma$-radiolysis however, the solutions did not give any measurable photon counts and hence did not contain particles with diameters $>6 \mathrm{~nm}$; which was the minimum resolution of the unit. The estimated volume average sizes were $\sim 49 \mathrm{~nm}$ for type I and $\sim 78 \mathrm{~nm}$ for type II solutions (table 1). Type II solutions clearly have larger particles as compared to type I solutions. It may be noted that the particle size measurements by DLS show larger particle diameters for a similar plasmon band spectrum, as compared to those by transmission electron microscopy (TEM) measurements ${ }^{8}$. This could be due to the fact that samples for TEM are evaporated to dryness and measured particle sizes are for bare metal particles. In contrast, DLS measures translational diffusion coefficient which is dependent on the solvation shell around the nanoparticle which possibly includes the stabilizer molecules and hence is expected to give higher values for the particle sizes. In another work, Yamamoto et al prepared SNP by the reduction of aqueous $\mathrm{AgNO}_{3}$ in presence of

Table 1. Volume average size of silver nanoparticles before and after irradiation with laser pulses to a circulating $50 \mathrm{ml}$ volume of SNP solution. Laser wavelength $=355 \mathrm{~nm}$; energy per pulse $=4 \mathrm{~mJ}$.

\begin{tabular}{lcccccc}
\hline & \multicolumn{2}{c}{ Before laser irradiation } & & \multicolumn{3}{c}{ After laser irradiation } \\
\cline { 2 - 3 } $\begin{array}{l}\text { Solutions } \\
{\left[\mathrm{Ag}^{+}\right]_{0}^{*}}\end{array}$ & $\begin{array}{c}\text { Vol. average } \\
\text { size } \\
(\mathrm{nm})\end{array}$ & $\begin{array}{c}\text { Width of } \\
\text { distribution } \\
(\mathrm{nm})\end{array}$ & & $\begin{array}{c}\text { Vol. average } \\
\text { size }^{\#} \\
(\mathrm{~nm})\end{array}$ & $\begin{array}{c}\text { Width of } \\
\text { distribution }^{\#} \\
(\mathrm{~nm})\end{array}$ & $\begin{array}{c}\text { Number } \\
\text { of } \\
\text { pulses }\end{array}$ \\
\hline $\begin{array}{l}\text { Type I } \\
(0 \cdot 1 \mathrm{mM})\end{array}$ & 68 & 51 & & 39 & 22 & 9000 \\
$\begin{array}{l}\text { Type I } \\
(0 \cdot 2 \mathrm{mM})\end{array}$ & 49 & 37 & & 29 & 28 & 9000 \\
$\begin{array}{l}\text { Type II } \\
(0 \cdot 5 \mathrm{mM})\end{array}$ & 78 & 75 & & 31 & 20 & 12000 \\
\hline
\end{tabular}

*Silver ion concentration before $\gamma$-irradiation; "accuracy of measurement in volume average size and width of distribution determinations in the present set of experiments is $\pm 5 \%$. 
$\mathrm{NaBH}_{4}{ }^{23}$. They measured the particle size using the DLS technique as $64 \mathrm{~nm}$ which is close to the DLS value for the particles prepared by us. However, in the work of Roberti et $a l^{8}$, the TEM values for the particle sizes were only $\sim 10 \mathrm{~nm}$, although their method for preparing the SNPs was similar to those of Yamamoto et al. The band widths of the SPB for the type I particles prepared by our method were comparable to those obtained by Roberti et al, suggesting similarities in the particle sizes as well.

\subsection{Transient changes upon flash photolysis}

Flash photolysis studies were carried out with type II solutions, having higher absorbance values as compared to the type I solutions, at the excitation wavelengths (namely 355 and $532 \mathrm{~nm}$, see figure 1). On excitation with $35 \mathrm{ps}$ pulses of $532 \mathrm{~nm}$, in the shoulder region of the SPB, a strong bleaching signal in the spectral region of 450-600 nm and a weak absorption band in the region 600-900 nm were seen at 'zero' ps delay (i.e. immediately after the pulse). There was no detectable bleach recovery on increasing the delay to 5 ns of the upper limit in our pump and probe technique (see figure $2 \mathrm{a}-1$ ). However, by employing the nanosecond adaptation of our detection system (see §2), it was observed that the bleaching was completely recovered at $100 \mathrm{~ns}$ after the $35 \mathrm{ps}$ pulse. The persistence of bleaching in the interval $5 \mathrm{~ns}<t<100 \mathrm{~ns}$ is an additional feature that

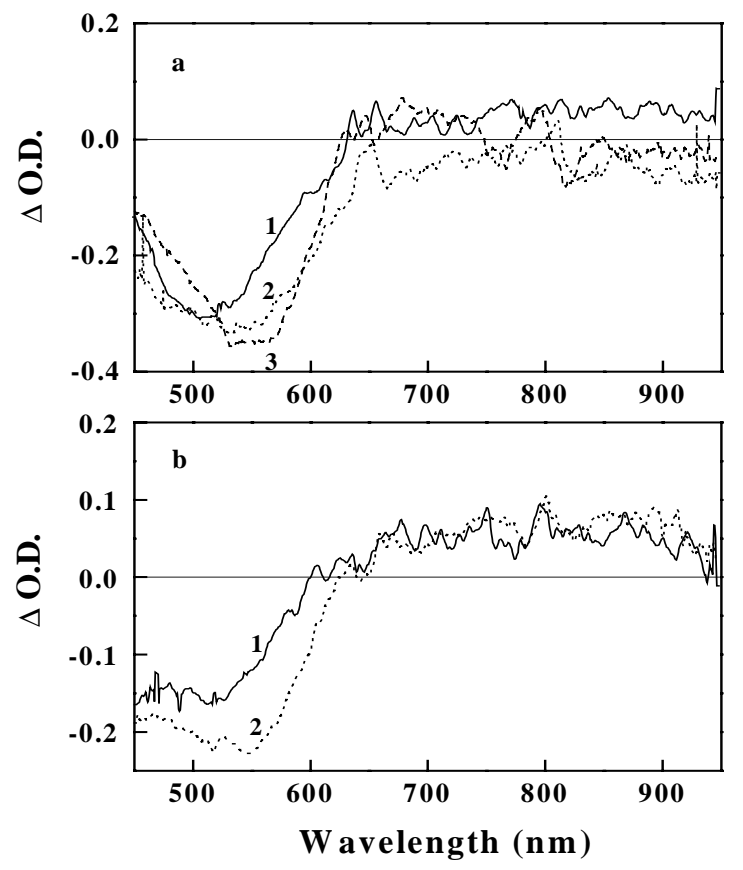

Figure 2. Transient bleaching/absorption signals between 'zero' to 5 ns delay after the photoexcitation of the silver nanoparticles with 35 ps laser pulse. (a) (1): Type II solutions, wavelength of excitation, $\lambda_{\mathrm{ex}}=532 \mathrm{~nm}$; energy $=4 \mathrm{~mJ} /$ pulse. (2): with $10 \%$ $(v / v)$ acetone addition; other conditions as in (1). (3): with $10 \%(v / v)$ 2-propanol addition; other conditions as in (1). (b) (1): Type II solutions, $\lambda_{\mathrm{ex}}=355 \mathrm{~nm}$; energy $=2 \mathrm{~mJ} /$ pulse. (2): with $10 \%(v / v)$ acetone addition; other conditions as in (1). 
was not observed by Roberti et al who found that the SPB excitations decayed with a lifetime of $\sim 40 \mathrm{ps}^{8}$. Also, the work of Ahmadi et al on gold colloids reported the time for bleach recovery, attributed to the relaxation by the phonon-cooling process, as $\geq 50 \mathrm{ps}^{9}$. Both studies were carried out with nanoparticles prepared without using any stabilizer. However, our results are in line with the more recent work by Kamat et al on the picosecond dynamics of silver nanoparticles prepared by reduction of $\mathrm{AgNO}_{3}$ by sodium citrate $^{10}$. In their study the bleach signal was found to rise in $\sim 90 \mathrm{ps}$ and recover in a few ns. The bleaching was found to be a monophotonic process. A weak absorption signal was also seen by them and is attributed to the generation of hydrated electron $\left(e^{-}{ }_{\text {aq }}\right)$ during photolysis.

Flash photolysis was also carried out with addition of acetone or 2-propanol to the type II solutions. The former reacts with $e^{-}$aq as well as the precursor state viz, the dry or quasifree electron ${ }^{24}$ while the latter is relatively inert. Addition of $10 \%$ acetone or $\mathrm{PrOH}$ decreases the absorption at $390 \mathrm{~nm}$ and increases that around the $550 \mathrm{~nm}$ shoulder in the surface plasmon band of type II solutions, as shown in figure 1c. On excitation with $532 \mathrm{~nm}$ pulses, the extent of the 450-600 nm bleaching increases at 0 ps delay, with an apparent reduction in the weak absorptions in the $600-900 \mathrm{~nm}$ region (figure $2 \mathrm{a}-2$
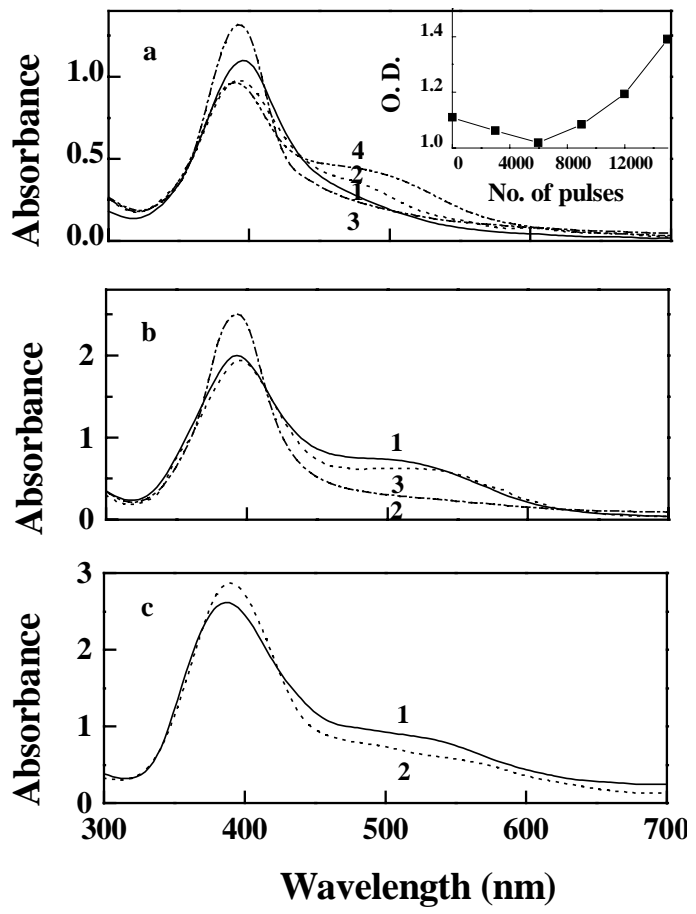

Figure 3. Changes in the optical absorption spectra of silver nanoparticles after photolysis of a circulating $50 \mathrm{ml}$ solution of the SNP with repetitive laser pulses. (a) Type I solutions with $\left[\mathrm{Ag}^{+}\right]_{0}=0 \cdot 2 \mathrm{mM}, \lambda_{\mathrm{ex}}=355 \mathrm{~nm}$, energy $=4 \mathrm{~mJ} /$ pulse. Number of pulses: (1) 0 , (2) 6000 , (3) 15000 , (4) 15000 (after $12 \mathrm{~h}$ aging). Inset: Variation of OD at $390 \mathrm{~nm}$ with the number of pulses. (b) Type II solutions $\left(\left[\mathrm{Ag}^{+}\right]_{0}=0.5 \mathrm{mM}\right)$, $\lambda_{\mathrm{ex}}=355 \mathrm{~nm}$, energy $=4 \mathrm{~mJ} /$ pulse. Number of pulses: (1) 0, (2) 12000, (3) 12000 (after $12 \mathrm{~h}$ aging). (c) Type II solutions $\left(\left[\mathrm{Ag}^{+}\right]_{0}=0.5 \mathrm{mM}\right), \quad \lambda_{\mathrm{ex}}=532 \mathrm{~nm}$, energy $=4 \mathrm{~mJ} /$ pulse. Number of pulses: (1) 0, (2) 600 . 
and 3). The maximum in the bleaching signal is red-shifted by about $20 \mathrm{~nm}$ in the solution containing $10 \%$ acetone and by about $30 \mathrm{~nm}$ in that containing $10 \% \mathrm{PrOH}$, as compared to solutions without acetone or $\mathrm{PrOH}$. The above shifts in the transient spectra could be attributed to the changes in the ground state spectra due to the presence of acetone or $\mathrm{PrOH}$ (see figure $1-\mathrm{b}$ and $\mathrm{c}$ ). However, the changes in the magnitude of bleaching and absorption signals brought about by $\mathrm{PrOH}$ or acetone could be regarded as small. It would appear that even at the molar concentrations employed, acetone did not interfere with the 'hot' electrons produced on the excitation of SPB.

Bleaching as well as absorption signals, similar to those described above, were observed when the type II solutions were irradiated with $355 \mathrm{~nm}$ pulses of $35 \mathrm{ps}$ duration (figure $2 b-1$ and 2 ). The similarity in the results obtained on photoexcitation by both $355 \mathrm{~nm}$ and $532 \mathrm{~nm}$ pulses support the fact that both $390 \mathrm{~nm}$ peak and $550 \mathrm{~nm}$ shoulder arise from the same plasmon band ${ }^{19,20}$ i.e. from the presence of predominantly one kind of particle (i.e. similar $\mathrm{a} / \mathrm{b}$ ratio) with different sizes in the type II solutions.

On excitation of type I solutions (displaying only the $390 \mathrm{~nm}$ band) by $355 \mathrm{~nm}$ laser pulses of 35 ps duration, only a very weak absorption signal in the region $500-900 \mathrm{~nm}$ was seen. It was not possible to study its decay due to small transient absorption intensities.

Picosecond flash photolysis experiments have been carried out earlier by Haase et al on CdS nanoparticles ${ }^{25}$, which showed photoelectron ejection in solutions. The electrons could be scavenged by known $e_{\text {aq }}^{-}$scavengers. More relevant to our present work are the observations by Kamat $e$ t al on the $355 \mathrm{~nm}$ laser excitations of aqueous solutions of silver nanoparticles. At laser intensities higher than $1 \mathrm{~mJ} /$ pulse, a weak absorption signal in the red region was obtained. This was attributed to the photoejection of electrons from the silver particles by a biphotonic process, resulting in the generation of $e_{\text {aq }}^{-}$and the observed optical absorption in the visible-near infrared region. In our studies, however, the magnitude of optical absorptions in the $600-900 \mathrm{~nm}$ remained nearly the same between 'zero' ps and $5 \mathrm{~ns}$ after $355 \mathrm{~nm}$ excitation ( $2 \mathrm{~mJ} / \mathrm{pulse})$, and was not diminished by the presence of molar concentrations of acetone, which is a very efficient $\mathrm{e}^{-}$aq scavenger (see figure $2 \mathrm{~b}$ ). It thus appears that the SPB excitations of the polyphosphate stabilized silver nanoparticles do not lead to a significant photoejection of electrons into the solvent across the particle-solvent interface. We prefer to understand the bleaching and absorption signals as due to the breaking up of the particles on SPB excitation, as described in the following section.

\subsection{Other effects of photolysis}

In addition to the transient optical changes following the SPB excitation of the SNPs; long-term alterations in the average sizes of the particles from both the type I and type II solutions have been studied. A $50 \mathrm{ml}$ solution was circulated through the flow cell which was irradiated with repeated laser pulses. On $355 \mathrm{~nm}$ excitation of the type I solutions $\left(1.45 \times 10^{8} \mathrm{~W} / \mathrm{cm}^{2}\right)$, broadening of the SPB was observed with a decrease in optical density (OD) at $390 \mathrm{~nm}$, up to 6000 laser pulses. At higher number of laser pulses, the OD at $390 \mathrm{~nm}$ increased beyond the pre-photolysis OD and the SPB became narrower. These changes are shown in figure $3 \mathrm{a}$. The inset of figure $3 \mathrm{a}$ shows the effect of increasing the number of $355 \mathrm{~nm}$ laser pulses on the OD at $390 \mathrm{~nm}$ in the SPB. It is seen that as the number of pulses increases, the OD at $390 \mathrm{~nm}$ decreases and then increases. These results indicate that on irradiation the particles first attain a critical size and then change over to 
smaller clusters, since a sharper SPB band is attributed to smaller clusters. Measurements by the DLS technique clearly showed that on laser photolysis of the solutions the volume average size of the particles decreased from $49 \mathrm{~nm}$ to $29 \mathrm{~nm}$ (9000 pulses, table 1). Further physical changes, apparently dependent on the initial $\mathrm{Ag}^{+}$ion concentration $\left(\left[\mathrm{Ag}^{+}\right]_{0}\right)$ were noticed on aging of the laser irradiated solutions. At $\left[\mathrm{Ag}^{+}\right]_{0}=0 \cdot 2 \mathrm{mM}$, overnight aging produced a colour change from yellow to reddish-brown, while with $\left[\mathrm{Ag}^{+}\right]_{0}=0.1 \mathrm{mM}$, such changes were not observed. It may be mentioned that the unphotolysed nanoparticle solutions themselves did not show any aging effect at either concentrations of the $\mathrm{Ag}^{+}$ion. When the photolysis was carried out using the conventional low intensity lamps (output at $\sim 350 \mathrm{~nm}$ ) in the Rayonet photochemical reactor, neither any sharpening of the SPB nor other changes comparable to those seen on the laser photolysis were observed. In these experiments, the total number of absorbed photons was kept nearly the same as in the laser irradiations. However, the light intensity was $\sim 10^{16}$ photons $\mathrm{cm}^{-2} \mathrm{~s}^{-1}$ as against $\sim 10^{15}$ photons $\mathrm{cm}^{-2}$ pulse of $35 \mathrm{ps}$ duration at $10 \mathrm{~Hz}$ (i.e. $\sim 3 \times 10^{26}$ photons $\mathrm{cm}^{-2} \mathrm{~s}^{-1}$ ) while employing the laser pulses. It was apparent from these results that the photolytic changes in the nanoparticles were intensity dependent.

Laser intensity effects on size redistribution of the silver clusters have been reported by Takami $e t$ al, who observe that the mode of distribution of silver particles shifts from 19 to $9 \mathrm{~nm}^{12}$. A possible mechanism proposed by the authors is as follows. The photoexcitation of SPB results in the desorption of $\mathrm{Ag}$ atoms/ions from the particle surface and the atom/ion desorption results in the size reduction of the SNPs. At the same time SNPs originally aggregated separate from each other. Kamat et al propose that on laser excitation, the SNP lose electrons by photoejection and a transient state of the particle is formed which is the precursor for complete fragmentation of the larger particles used in their work. They also propose that the particles become charged on laser irradiation and once sufficient charge accumulates on the particle, it breaks ${ }^{10}$. Our results, however, do not support the photoejection of electrons, as the experiments with acetone, a good scavenger of electrons, would have shown different results. However, in the presence of a stabilizer like polyphosphate, the fragmented particles reassemble to give new particles with different sizes, as given below:

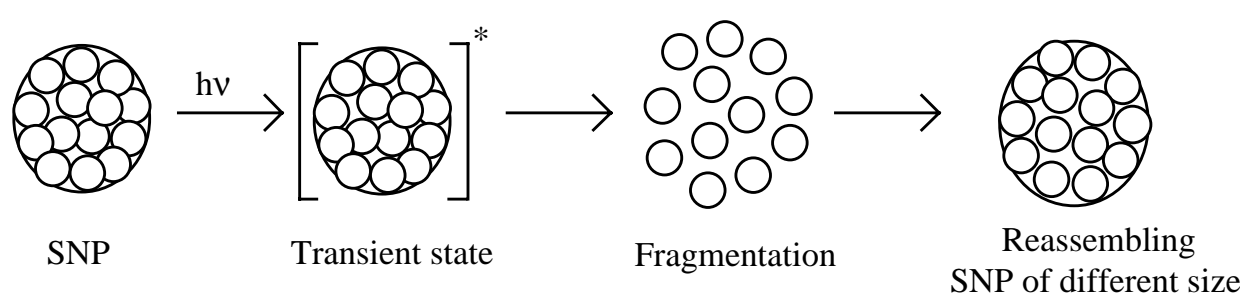

The particle fragments could be of such dimensions/nuclearity that they would not possess the characteristic surface plasmon band of silver nanoparticles. Therefore, during the period that the particle remains fragmented, bleaching of the plasmon band would result. The bleaching observed by us between 'zero' ps and $\leq 5 \mathrm{~ns}$ could represent this period. The small absorption signals in the $600-900 \mathrm{~nm}$ could also be the net effect of the fragmentation. However, the duration $t$ where $5 \mathrm{~ns}<t<100 \mathrm{~ns}$ could represent the slow reassembling process leading to the recovery of the plasmon band.

The metal clusters are held together with moderate to strong binding energies from $0 \cdot 5-3 \mathrm{eV}^{26}$. Due to the multiphotonic nature of the excitation, the energy available with 
the nanoparticle exceeds the binding energy resulting in the fragmentation of the nanoparticle as observed by us. However, the fragments are unstable as compared to the nanoparticles. Therefore, the fragments would reassemble to give another set of nanoparticles with different size distribution.

As already mentioned, continuous irradiation in the photochemical reactor does not result in sharpening of the SPB or similar changes at the same number of total absorbed photons but at drastically lower fluences, (difference of $\sim 10^{10}$ times). It can be seen that either biphotonic or multiphotonic effects are responsible for the observed particle fragmentation on laser irradiation. This observation is true for both 355 and $532 \mathrm{~nm}$ irradiation with laser pulses. Kamat et al found that plasmon bleach was dependent on intensity at intensities $<1 \mathrm{~mJ} /$ pulse while it became independent of intensity above this energy due to saturation effects. The transient absorption was found by them to obey quadratic dependence on the laser dose. They concluded that the primary process to break a nanocluster was biphotonic ${ }^{10}$. Our results from low intensity experiments also show that the fragmentation of SNP would involve more than one photon; with the energy input adequate to overcome the binding energy of the nanoparticles.

The influence of laser irradiation on gold nanoparticles has been recently reported by Kimura and coworkers and they observed that the stability of nanoparticles increased after laser irradiation ${ }^{27,28}$. In our work, when type II solutions were irradiated continually with $355 \mathrm{~nm}$ or $532 \mathrm{~nm}$ laser pulses, sharpening of $390 \mathrm{~nm}$ band was seen. Furthermore, the OD of the shoulder band around $550 \mathrm{~nm}$ was found to decrease and that of $390 \mathrm{~nm}$ band increase (figures $3 \mathrm{~b}$ and $\mathrm{c}$ ). Simultaneously, the reddish-brown colour of the solution changed to yellow. These results would imply progressive conversion of the particles from type II to type I during the laser photolysis. In addition, the photolysis was found to decrease the volume average particle size from $\sim 78 \mathrm{~nm}$ to $31 \mathrm{~nm}$ (table 1). Further changes accompanying the aging of the photolysed solutions were observed in these solutions also. On keeping the solutions overnight, the colour of the solution was found to revert from yellow to reddish-brown, and the spectrum corresponded to that typical of type II solution. Some of the intermediate stages in these post-irradiation changes might involve the familiar 'Ostwald-ripening' 28.

It is probable that the polyphosphate stabilizer employed in the present work had a definite role in producing the type II solutions, leading to the observed transient as well as post-photolysis changes. Radiolytic preparation of the SNP without the stabilizer did not yield clusters displaying the shoulder band and having the desired stability for further experimentation. The preparations, where sodium dodecyl sulphate (SDS) was used as the stabilizer, displayed yellow colour; however, the ODs beyond $450 \mathrm{~nm}$, especially in the shoulder region were much smaller as compared to the SNP stabilization with polyphosphate.

\section{Conclusions}

Using the technique of $\gamma$-radiolysis, two types of silver nanoparticles (SNP) were generated, one with a near-Gaussian surface plasmon band centred at $\sim 390 \mathrm{~nm}$ (type I) and another with a similar band and an additional shoulder at $550 \mathrm{~nm}$ (type II). On flash photolysis by $35 \mathrm{ps}, 532 \mathrm{~nm}$ pulses, the latter showed transient bleaching signal in the $450-600 \mathrm{~nm}$ region. The signal was found to last longer than $5 \mathrm{~ns}$. By separate nanosecond flash photolysis experiments, the lifetime for the relaxation process giving rise to bleach recovery was estimated to be less than $100 \mathrm{~ns}$. A small positive transient 
absorption signal was also seen at $>600 \mathrm{~nm}$ for the type II solutions. However, the absorption in this region could not be attributed to photoejection of electrons from the phosphate-stabilized silver nanoparticles. Photolysis of the nanoparticles with repetitive $355 \mathrm{~nm}$ and $532 \mathrm{~nm}$ laser pulses was found to narrow the surface plasmon band and decrease the particle size. It is proposed that on laser irradiation the SNP breaks down to smaller fragments which reassemble to give particles of new dimensions.

\section{Acknowledgement}

We thank Mr P A Hassan for carrying out the particle size measurements using the DLS technique.

\section{References}

1. Kreibig U 1974 J. Phys. F4 999

2. Kerker M 1969 The scattering of light and other electromagnetic radiation (New York: Academic Press)

3. Huffman D R 1988 Optical effects associated with small particles (eds) P W Barber and R K Chang (World Scientific: Singapore)

4. Mulvaney P 1996 Langmuir 12788

5. Mulvaney P, Linnert T and Henglein A 1991 J. Phys. Chem. 957843

6. Mulvaney P, Linnert T and Henglein A 1991 Ber. Bunsen-Ges. Phys. Chem. 95838

7. Linnert T, Mulvaney P, Henglein A and Weller A 1990 J. Am. Chem. Soc. 1124657

8. Roberti T W, Smith B A and Zhang J Z 1995 J. Chem. Phys. 1023860

9. Ahmadi T S, Logunov S L and El-Sayed M A 1996 J. Phys. Chem. 1008053

10. Kamat P V, Flumiani M and Hartland G V 1998 J. Phys. Chem. 1023123

11. Groeneveld R H M, Sprik R and Lagendrigk A 1990 Phys. Rev. Lett. 64784

12. Takami A, Yamada H, Nakano K and Koda S 1996 Jpn. J. Appl. Phys. L35 781

13. Kapoor S 1998 Langmuir 141021

14. Henglein A 1977 Ber. Bunsen-Ges. Phys. Chem. 81556

15. Tausch-Treml R, Henglein A and Lilie J 1978 Ber. Bunsen-Ges. Phys. Chem. 821335

16. Henglein A and Tausch-Treml R 1981 J. Colloid Interface Sci. 8084

17. Henglein A 1989 Chem. Phys. Lett. 154473

18. Palit D K, Sapre A V and Mittal J P 1992 Indian J. Chem. A\&B31 F42

19. Palit D K, Sapre A V, Mittal J P and Rao C N R 1992 Chem. Phys. Lett. 1951

20. Ghosh H N, Sapre A V, Palit D K and Mittal J P 1997 J. Phys. Chem. 1012315

21. Skillman D C and Berry C R 1968 J. Chem. Phys. 483297

22. Bohren C F and Huffman D R 1983 Absorption and scattering of light by small particles (New York: John Wiley)

23. Yamamoto H, Maeda Y and Kitand H 1997 J. Phys. Chem. 1016855

24. Chase W J and Hunt J W 1975 J. Phys. Chem. 792835

25. Haase M, Weller H and Henglein A 1988 J. Phys. Chem. 924708

26. Jortner J, Scharf D and Landman U 1987 Elemental and molecular clusters (eds) G Benedek, T P Martin and G Pacchioni (Berlin: Springer-Verlag) p. 148

27. Takeuchi Y, Ida T and Kimura K 1997 J. Phys. Chem. B101 1322

28. Satoh N, Hasegawa H, Tsujii K and Kimura K 1994 J. Phys. Chem. 982143 